\title{
FUNÇÃO FONATÓRIA EM PACIENTES COM DOENÇA DE PARKINSON: USO DE INSTRUMENTO DE SOPRO
}

\author{
Phonatory function in Parkinson's disease patients: \\ use of wind instrument
}

\author{
Juliana de Caldas Rosa ${ }^{(1)}$, Carla Aparecida Cielo ${ }^{(2)}$, Cláudio Cechella ${ }^{(3)}$
}

\begin{abstract}
RESUMO
Objetivo: verificar o efeito da terapia com instrumento de sopro na função fonatória de pacientes com doença de Parkinson, com enfoque nas avaliações laríngeas, acústicas, respiratórias e de intensidade vocal. Métodos: esta pesquisa é um estudo de caso coletivo e prospectivo. Dois sujeitos foram submetidos a exame videolaringoscópico, avaliação da função respiratória, gravação de amostras da voz, medição da intensidade vocal e terapia. Para a análise acústica foi utilizado o programa Multidimensional Voice Program advanced. A análise dos dados foi ponderada, uma vez que a pesquisa é um estudo de caso coletivo. Resultados: os resultados mostraram que as modificações respiratórias, acústicas e de intensidade vocal foram similares e positivas nos dois sujeitos estudados. Conclusão: a terapia realizada com o instrumento de sopro pode proporcionar maior eficiência na adução glótica, e melhor movimentação e controle respiratório, propiciando o uso mais efetivo do ar para fonação, com melhora da qualidade vocal, expressa pela redução das medidas de ruído, maior estabilidade fonatória e aumento da intensidade vocal.
\end{abstract}

DESCRITORES: Doença de Parkinson; Fonoterapia; Voz

\section{INTRODUÇÃO}

A doença de Parkinson (DP) é uma afecção neurológica progressiva, cujas manifestações clínicas incluem tremor, rigidez, acinesia e alteração dos reflexos posturais ${ }^{1}$. As alterações fonoarticulatórias na DP caracterizam a disartria hipocinética, com os sintomas manifestando-se na voz e na fala. Estas manifestações podem ser observadas ao longo de todo o período da doença, afetando os vários sis-

(1) Fonoaudióloga; Serviço de Fonoaudiologia, Psicopedagogia e Psicologia Escolar da Prefeitura Municipal de Gaspar, Gaspar, SC; Integrante do grupo de pesquisa em Voz da Universidade Federal de Santa Maria, UFSM, Santa Maria, RS.

(2) Fonoaudióloga; Professora Adjunto de Graduação do Departamento de Fonoaudiologia e do Programa de PósGraduação em Distúrbios da Comunicação Humana da Universidade Federal de Santa Maria, UFSM, Santa Maria, RS; Doutora em Linguística Aplicada pela Pontifícia Universidade Católica do Rio Grande do Sul.

(3) Neuropediatra; Professor Assistente do Departamento de Fonoaudiologia da Universidade Federal de Santa Maria, UFSM, Santa Maria, RS; Mestre em Medicina - Área de Concentração Neurologia pela Universidade Federal do Rio de Janeiro.

Conflito de interesse: INEXISTENTE temas envolvidos na função fonatória: respiratório, articulatório e laríngeo ${ }^{2}$. Os fatores que determinam as modificações e a severidade dos sintomas, relacionam-se ao aumento da rigidez e da bradicinesia, que afeta também a musculatura laríngea e respiratória.

O distúrbio vocal normalmente está associado à hipofunção laríngea, com redução da intensidade vocal $^{1,2}$. A intensidade vocal diminuída está relacionada à menor pressão subglótica, devido ao fechamento glótico incompleto, geralmente encontrado em pacientes parkinsonianos ${ }^{1,3-5}$, seja pela presença de fenda à fonação ${ }^{6}$, pelo arqueamento de pregas vocais ${ }^{3-5}$, ou ainda pelo aumento de rigidez da musculatura adutora ${ }^{7}$.

$\mathrm{Na}$ diminuição da intensidade vocal estão implicadas, também, as modificações que ocorrem no sistema respiratório, em função da rigidez e bradicinesia, uma vez que afetam a musculatura respiratória. Há a diminuição da mobilidade torácica, com redução da capacidade vital, da capacidade inspiratória ${ }^{1}$ e dos tempos máximos de fonação. Há, também, interferência no controle da intensidade vocal, devido ao menor suporte respiratório e redução da 
pressão expiratória ${ }^{1}$, responsáveis pelas modificações da pressão aérea subglótica ${ }^{8}$.

O fechamento ineficiente das pregas vocais resulta em escape de ar, ocasionando modificações na qualidade da voz, que pode se apresentar com características de soprosidade ${ }^{2,3,5,7}$, rouquidão ${ }^{2,3}$ e aspereza ${ }^{5}$. O tremor vocal também pode estar presente $^{3}$. Na análise acústica das vozes de pacientes com DP, correlacionadas às modificações acústico-perceptivas, observam-se valores elevados de jitter ${ }^{1,9}$ e de shimmer, e diminuição dos valores da relação harmônico-ruído ${ }^{1}$.

Estudos mostram que, durante a execução de tons musicais em instrumentos de sopro, a laringe atua no suporte e no controle do fluxo de ar 10-12. As pregas vocais fazem um movimento de adução, mantendo-se numa posição predominantemente intermediária durante a produção do som, principalmente na sustentação de notas em forte intensidade ${ }^{10}$. Esta aproximação das pregas vocais foi notada durante a execução com diferentes tipos de instrumentos de sopro ${ }^{10-13}$.

Tocar um instrumento de sopro é uma atividade intensa para o sistema respiratório, que demanda tanto força dos músculos respiratórios, quanto controle da respiração ${ }^{14}$. O efeito do treinamento respiratório no aumento da pressão expiratória é similar independentemente do instrumento de sopro utilizado $^{8}$. Desse modo, o treinamento respiratório em pacientes parkinsonianos poderia contribuir para maior mobilização torácico-abdominal e para o aumento da força muscular, com melhora da função respiratória, bem como para o aumento da intensidade vocal.

O fechamento glótico incompleto e a redução da expansibilidade torácica, encontrados em pacientes com DP, afetam a intensidade vocal e interferem negativamente na comunicação destes indivíduos. $\mathrm{Na}$ hipótese de que o uso de instrumentos de sopro poderia contribuir para melhorar a função fonatória, proporcionando um trabalho muscular de adução e resistência das pregas vocais, e maior controle da função respiratória.

Este estudo foi realizado com o objetivo de verificar o efeito da terapia fonoaudiológica com instrumento de sopro na função fonatória de pacientes com DP.

\section{MÉTODOS}

Esta pesquisa é um estudo de caso coletivo, que consiste em analisar conjuntamente alguns casos para investigar um dado fenômeno, selecionados por manifestarem ou não alguma característica comum, sendo que, a partir de seu estudo poderá haver melhor compreensão, ou mesmo melhor teorização, sobre um conjunto ainda maior de casos ${ }^{15}$. A pesquisa foi de campo, longitudinal, não-experimental, quanti-qualitativa e prospectiva. Os critérios para sua realização foram embasados na norma 196/96 (CONEP), com a assinatura do termo de consentimento livre e esclarecido, pelos voluntários participantes deste estudo.

A seleção dos sujeitos incluídos nesta pesquisa foi feita por meio de contato pessoal. Foram utilizados os seguintes critérios de inclusão: ter idade entre 50 e 80 anos; ter diagnóstico médico de doença de Parkinson; estar usando medicação anti-parkinsoniana e não modificar a dosagem do mesmo durante o período do tratamento. Os critérios de exclusão foram: apresentar comprometimento das condições motoras manuais; não possuir liberação médica para o trabalho com esforço respiratório; apresentar características laríngeas diferentes daquelas típicas da doença de Parkinson e ou da presbilaringe e realizar ou ter realizado tratamento fonoaudiológico e ou fisioterapêutico nos últimos quatro meses.

Em um estudo ${ }^{2}$, verificou-se que a função fonatória apresentou-se com características semelhantes em todos os estágios da doença de Parkinson, não se associando com a duração da doença. E, em outro estudo ${ }^{3}$, não foi encontrada correlação significativa entre o estágio da doença e o tempo de aparecimento dos sintomas vocais. Desta forma, não foi adotada na pesquisa a delimitação do estágio da doença.

Participaram do estudo dois sujeitos, sendo um do sexo masculino e um do sexo feminino, com 52 e 66 anos, respectivamente. Ambos foram submetidos aos seguintes procedimentos avaliativos: anamnese, exame videolaringoscópico, avaliação da função respiratória, gravação de amostra da voz, medição da intensidade vocal e terapia.

O exame videolaringoscópico foi realizado com um laringoscópio de fibra óptica de tubo rígido, antes e após o término do período total da terapia, pelo mesmo médico otorrinolaringologista. Os aspectos laríngeos analisados foram: presença e tipo de fenda glótica durante a emissão; presença de arqueamento de pregas vocais; presença e grau de participação do vestíbulo laríngeo durante a fonação; presença de lesões e de sinais de refluxo gastroesofágico; características da mucosa e de mobilidade das pregas vocais.

A função respiratória foi avaliada por meio do espirômetro seco (FIMI-ITÁ), e de medidas de tempo máximo de fonação registradas com a utilização de um cronômetro (Nokia 3310). Os aspectos respiratórios medidos, antes e após o período total de tratamento, foram: capacidade vital (maior de três medidas da expiração, com o uso do 
espirômetro, após inspiração máxima); tempos máximos de fonação (TMF) para as vogais /a/, /i/ e /u/ (média de três medidas de cada vogal sustentada pelo tempo máximo que o indivíduo conseguiu, após inspiração máxima e emissão em tom e intensidade habituais); e relação $s / z$ (foi calculada a média de três medidas de cada consoante, sustentada pelo tempo máximo após inspiração máxima, e computado o valor da relação s/z).

A gravação da voz foi realizada antes e após o período total de tratamento, em ambiente com baixo nível de ruído ambiental $(<$ que $50 \mathrm{~dB}){ }^{1}$. Um gravador digital (marca Maxmux - MP4 player) foi colocado a uma distância de quatro centímetros da boca do sujeito ${ }^{16}$, que emitiu a vogal /a/ sustentada, no seu tom e intensidade habituais.

A análise dos parâmetros acústicos da voz foi realizada utilizando-se o programa Multidimensional Voice Program (MDVPadvanced), da Kay Elemetrics Corp. As medidas acústicas avaliadas foram: Average Fundamental Frequency (fO), Standard Deviation of fo (STD), fO-Tremor Frequency (Fftr), Amplitude Tremor Frequency (Fatr), fo-Tremor Intensity Index (FTRI), Amplitude Tremor Intensity Index (ATRI), Absolute Jitter (Jita), Jitter Percent (Jitt), Relative Average Perturbation (RAP), Pitch Perturbation Quotient (PPQ), Smoothed Pitch Perturbation Quotient (sPPQ), Fundamental Frequency Variation (vfO), Shimmer in $d B$ (ShdB), Shimmer Percent (Shim), Amplitude Perturbation Quotient (APQ), Smoothed Amplitude Perturbation Quotient (sAPQ), Peak-to-Peak Amplitude Variation $(v A m)$, Noise to Harmonic Ratio (NHR), Voice Turbulence Index (VTI), Soft Phonation Index (SPI), Degree of Voice Breaks (DVB), Degree of Sub-harmonics (DSH), Degree of Voiceless (DUV), Number of Voice Breaks (NVB), Number of Subharmonic Segments (NSH) e Number of Unvoiced Segments (NUV).

Foram analisadas duas amostras vocais de cada sujeito, obtidas antes e também ao final do período total de terapia, utilizando-se a média dos resultados acústicos das mesmas ${ }^{17}$. As amostras analisadas estavam no formato PCM, $44.1 \mathrm{kHz}$ e 16-bit, tendo sido excluídos o início e o final da gravação.

Para a medida da intensidade vocal, foi usado um decibelímetro (RadioShack Digital Sound Level Meter - 33-2055) posicionado à distância de um metro, em ambiente com baixo nível de ruído ambiental $(<50 \mathrm{~dB})$, procedendo-se à emissão da vogal /a/ sustentada, em intensidade vocal habitual e, num segundo momento, em forte intensidade.

A terapia foi realizada por 12 semanas, com sessões semanais de 40 minutos. Cada sessão foi composta por: preparação corporal (posicionamento e postura) e manual (posicionamento dos dedos no instrumento de sopro), e exercícios de sopro com instrumento (flauta doce).

Foram realizadas oito séries de exercícios, compostas de oito repetições, estipulando-se um período de repouso passivo de um minuto entre cada série ${ }^{18}$. A intensidade da execução do sopro foi determinada, baseando-se no estudo, no qual os autores verificaram que a adução glótica é maior na condição de execução do sopro em forte intensidade para notas sustentadas ${ }^{10}$, e no estudo onde foi verificado que exercícios expiratórios específicos, realizados em intensidade elevada e com poucas repetições, aumentaram a pressão expiratória, independentemente do instrumento usado ${ }^{8}$.

Todos os exercícios de sopro foram realizados em intensidade forte, e foram distribuídos na sessão da seguinte forma: notas sustentadas, uma em cada expiração, em escala ascendente (série 1) e descendente (série 2); notas sustentadas, duas em cada expiração, em escala ascendente (série 3) e descendente (série 4); notas sustentadas, três em cada expiração, em escala ascendente (série 5) e descendente (série 6); e notas sustentadas, uma em cada expiração, em escala ascendente (série 7) e descendente (série 8).

Além da sessão semanal de terapia, os exercícios de sopro com o instrumento foram realizados pelo próprio sujeito em seu domicílio, na frequência de quatro vezes por semana, uma vez por dia, com duração de 10 minutos cada vez ${ }^{18}$.

O tempo entre a ingestão da medicação e os exercícios de sopro foi de 90 minutos, estabelecido em função do tempo em que a medicação atinge o seu pico de disponibilidade plasmática, relacionado na bula dos próprios medicamentos, estando o paciente sempre em estado "on".

O projeto foi registrado no Gabinete de Projetos do Centro de Ciências da Saúde (n020890) e aprovado pelo Comitê de Ética em Pesquisa da instituição de origem (n-23081.008439/2007-16).

A análise dos dados foi ponderada, considerando-se que se adotou, nesta pesquisa, o estudo de caso coletivo ${ }^{15}$.

\section{RESULTADOS}

Sujeito 1: sexo masculino, 52 anos, $1,70 \mathrm{~m}$ de altura. Medicação utilizada: levodopa. Como o paciente estava debilitado e não havia tomado sua medicação, na ocasião da primeira avaliação da função respiratória e da gravação de voz, estas foram realizadas com o paciente deitado na cama, com o tronco semi-ereto apoiado no travesseiro. Em razão disso, na reavaliação pós-tratamento, as mesmas condições foram reproduzidas, estando o paciente na mesma postura e sem o efeito medicamentoso. 
No entanto, na avaliação videolaringoscópica, o paciente estava em estado "on", no momento das duas avaliações (pré e pós-tratamento).

Sujeito 2: sexo feminino, 66 anos, 1,53m de altura. Medicação utilizada: levodopa. Na ocasião de todas as avaliações, a paciente estava em estado "on".

Os resultados das avaliações videolaringoscópica, respiratória, vocal acústica, e da intensidade vocal, pré e pós-terapia, dos pacientes, encontramse nas Tabelas 1, 2, 3, 4, respectivamente.

\section{DISCUSSÃO}

Na primeira avaliação (Tabela 1), a presença de fenda vocal com arqueamento de pregas vocais, encontrada no sujeito 1 , e a possível redução da resistência glótica do sujeito 2 podem ter interferido no padrão vibratório das pregas vocais ${ }^{6} \mathrm{e}$ na produção vocal, em função da falta de eficiência no fechamento glótico. Desta forma, as modificações na qualidade vocal, pelo escape de ar à fonação, mostradas pela análise acústica, e a menor intensidade vocal apresentada pelos sujeitos no início da terapia, podem estar relacionadas às alterações do fechamento glótico. Soma-se a isso, o aumento de rigidez (perda de plasticidade muscular) da musculatura adutora, normalmente presente na $\mathrm{DP}^{7}$, que diminui a amplitude dos movimentos das pregas vocais, interferindo na sua eficiência adutora.

Após a terapia, observou-se que o exame videolaringoscópico (Tabela 1) apontou mudança da fenda fusiforme para fenda paralela, no sujeito 1 , com eliminação do arqueamento de pregas vocais. A fenda fusiforme está associada às disfonias hipocinéticas, nas quais há deficiência da atividade da musculatura glótica adutora. Na fenda paralela, a falta de coaptação glótica é observada de maneira uniforme ao longo da borda livre das pregas vocais e pode estar relacionada a uma astenia vocal leve 19. A modificação ocorrida pode indicar que houve uma melhora do condicionamento muscular das pregas vocais, favorecendo uma melhor adução. Já, no sujeito 2, não houve modificações visíveis neste exame, mantendo-se o fechamento glótico completo, sem sinais de arqueamento de pregas vocais. Porém, analisando-se os demais parâmetros vocais pesquisados e discutidos posteriormente, acreditase que o padrão de fechamento glótico passou a ser realizado com maior firmeza muscular, o que auxiliou a redução do escape aéreo.

$\mathrm{Na}$ análise dos parâmetros respiratórios (Tabela 2), o aumento verificado na capacidade vital deve ser considerado como resultado da maior mobilização tóraco-abdominal devido ao treino respiratório. Este, por proporcionar também melhor suporte respiratório e controle do fluxo de ar ${ }^{12}$, acaba por

\section{Tabela 1 - Resultados do exame videolaringoscópico para os aspectos laríngeos}

\begin{tabular}{lcc|cc}
\hline \multirow{2}{*}{ Aspectos laríngeos } & \multicolumn{2}{c|}{ Sujeito 1 } & \multicolumn{2}{c}{ Sujeito 2 } \\
& pré- tratamento & pós- tratamento & pré- tratamento & pós- tratamento \\
\hline Configuração glótica & fenda fusiforme & fenda paralela & fechamento glótico & fechamento glótico \\
& presente & ausente & ausente & completo \\
Arqueamento de ppvv & ausente & ausente & ausente & ausente \\
Participação supraglótica & ausente & ausente & ausente & ausente \\
Presença de lesões & ausente & ausente & ausente & ausente \\
Sinais de RGE & sem particularidades & sem particularidades & sem particularidades & sem particularidades \\
Mucosa de ppvv & sem particularidades & sem particularidades & sem particularidades & sem particularidades \\
Mobilidade de ppvv & & & &
\end{tabular}

Tabela 2 - Resultados da avaliação da função respiratória, dos dois sujeitos

\begin{tabular}{|c|c|c|c|c|c|c|c|c|}
\hline \multirow[b]{2}{*}{ Parâmetro } & \multicolumn{4}{|c|}{ Sujeito 1} & \multicolumn{4}{|c|}{ Sujeito 2} \\
\hline & $\begin{array}{c}\text { pré- } \\
\text { tratamento }\end{array}$ & $\begin{array}{c}\text { pós- } \\
\text { tratamento }\end{array}$ & Variação & $\begin{array}{c}\text { Valor } \\
\text { esperado }\end{array}$ & $\begin{array}{c}\text { pré- } \\
\text { tratamento }\end{array}$ & $\begin{array}{c}\text { pós- } \\
\text { tratamento }\end{array}$ & Variação & $\begin{array}{c}\text { Valor } \\
\text { esperado }\end{array}$ \\
\hline Capacidade Vital (ml) & 2500 & 3000 & $20,00 \%$ & $3300^{*}$ & 1800 & 2100 & $16,67 \%$ & $2200^{\dagger}$ \\
\hline TMF média vogais (s) & 9,1 & 10,79 & $18,57 \%$ & $20^{\S}$ & 11,71 & 12,93 & $10,42 \%$ & $14^{\|}$ \\
\hline $\mathrm{TMF} / \mathrm{a} /(\mathrm{s})$ & 10,48 & 12,87 & $22,80 \%$ & $20^{\circ}$ & 11,28 & 17,43 & $54,52 \%$ & $14^{\prime \prime}$ \\
\hline Relação s/z & 0,92 & 0,76 & $-0,16$ & $0,8-1,2$ & 1,12 & 0,73 & $-0,39$ & $0,8-1,2$ \\
\hline
\end{tabular}

* valor de normalidade para homens com 1,70m de altura ${ }^{19}$

$\dagger$ media dos valores de normalidade para mulheres com altura entre 1,50 e 1,55m ${ }^{19}$

$\S$ valor mínimo de normalidade para homens ${ }^{19}$

|| valor mínimo de normalidade para mulheres ${ }^{19}$

TMF - tempo máximo de fonação 
refletir-se no aumento da média dos tempos máximos de fonação. Estes últimos são medidas que indicam, além do controle do fluxo de ar, o controle das forças mioelásticas da laringe, e avaliam a eficiência glótica ${ }^{19}$. Desta forma, o aumento dos tempos máximos de fonação, juntamente com a redução da relação s/z, apontou melhora do fechamento e/ou firmeza glótica.

Pode-se notar que, apesar da capacidade vital ainda estar abaixo do esperado para a altura, em ambos os sujeitos, ela se encontrou dentro dos valores mínimos esperados de $2200 \mathrm{ml}$ para homens e de $2100 \mathrm{ml}$ para mulheres ${ }^{19}$. E, embora os tempos máximos de fonação, considerando-se a média das vogais, estejam abaixo do esperado para o sexo, houve um aumento superior a $10 \%$.

Além disso, o treino respiratório contribuiu para o fortalecimento muscular, com melhora da função respiratória, aumentando a pressão expiratória ${ }^{8}$, e modificando a pressão subglótica, um dos mecanismos responsáveis pela variação da intensidade vocal. Estas modificações são importantes, uma vez que o incremento da pressão subglótica pode promover a melhora do fechamento glótico e evitar irregularidades vibratórias em pacientes com DP ${ }^{7}$.

A melhora do controle pneumofonoarticulatório contribui para a estabilidade durante a emissão sustentada, e, em associação a um melhor padrão vibratório e à melhor adução, proporciona maior aproveitamento do ar para sonorização, reduzindo o escape de ar, o que pode ser verificado nos resultados da análise acústica, que mostrou redução nas várias medidas relacionadas ao ruído como: Jita, Jitt, RAP, PPQ, sPPQ, ShdB, Shim, APQ, sAPQ, NHR (Tabela 3).

$\mathrm{Na}$ análise acústica de ambos os sujeitos (Tabela 3), tanto as medidas de jitter (Jita, Jitt, RAP, PPQ, SPPQ), quanto as relacionadas ao shimmer (ShdB, Shim, APQ, sAPQ), apresentaram redução do seu valor, sugerindo que houve maior periodicidade vibratória e redução de ruído à emissão, e, provavelmente, diminuição dos sinais de rouquidão e soprosidade nas características vocais dos sujeitos. O que pode ser reforçado pela redução do valor da medida de SPI, que sugere melhor coaptação glótica e maior firmeza de fechamento, com menor escape de $\operatorname{ar}^{3}$.

A diminuição das medidas correlacionadas ao shimmer evidencia maior controle glótico à saída do ar como resultado da terapia aplicada. A maior variação observada no sujeito 1 pode estar relacionada à mudança no fechamento glótico, de uma fenda fusiforme para uma paralela. Verifica-se que as médias finais, das medidas relacionadas a este parâmetro,

Tabela 3 - Resultados da análise acústica, realizada pelo MDVP, dos dois sujeitos

\begin{tabular}{|c|c|c|c|c|c|c|c|c|c|c|c|c|}
\hline \multirow[b]{2}{*}{ Parâmetro } & \multicolumn{6}{|c|}{ Sujeito 1} & \multicolumn{6}{|c|}{ Sujeito 2} \\
\hline & $\begin{array}{c}\text { Média } \\
\text { pré- } \\
\text { tratamento }\end{array}$ & $\begin{array}{l}\text { Média pós- } \\
\text { tratamento }\end{array}$ & $\begin{array}{l}\text { Variação } \\
\text { absoluta }\end{array}$ & $\begin{array}{l}\text { Variação } \\
\text { absoluta\% }\end{array}$ & $\begin{array}{l}\text { Variação\% } \\
\text { em relação } \\
\text { ao limiar* }\end{array}$ & Limiar & $\begin{array}{c}\text { Média } \\
\text { pré- } \\
\text { tratamento }\end{array}$ & $\begin{array}{l}\text { Média pós- } \\
\text { tratamento }\end{array}$ & $\begin{array}{l}\text { Variação } \\
\text { absoluta }\end{array}$ & $\begin{array}{l}\text { Variação } \\
\text { absoluta\% }\end{array}$ & $\begin{array}{l}\text { Variação\% } \\
\text { em relação } \\
\text { ao limiar* }\end{array}$ & Limiar \\
\hline fo $(\mathrm{Hz})$ & 117.694 & 105.536 & -12.158 & $-10,33 \%$ & $-10,33 \%$ & & 179.395 & 171.129 & -8.266 & $-4,61 \%$ & $-4,61 \%$ & \\
\hline STD $(\mathrm{Hz})$ & 17.216 & 3.245 & -13.971 & $-81,15 \%$ & $-88,05 \%$ & $1.349^{\dagger}$ & 7.123 & 2.487 & -4.636 & $-65,08 \%$ & $-105,36 \%$ & $2.722^{\dagger}$ \\
\hline $\mathrm{Fftr}(\mathrm{Hz})$ & 3.450 & 5.923 & 2.473 & $71,68 \%$ & $71,68 \%$ & $8.000^{\S}$ & 4.650 & & -4.650 & $-100,00 \%$ & $-100,00 \%$ & $8.000^{\S}$ \\
\hline Fatr $(\mathrm{Hz})$ & 2.921 & 3.485 & 564 & $19,31 \%$ & $19,31 \%$ & $8.000^{\S}$ & 2.986 & 1.754 & -1.232 & $-41,26 \%$ & $-58,74 \%$ & $8.000^{\S}$ \\
\hline Jita ( $\mu \mathrm{s})$ & 552.779 & 182.450 & -370.329 & $-66,99 \%$ & $-78,86 \%$ & 83.200 & 156.480 & 48.606 & -107.874 & $-68,94 \%$ & $-147,21 \%$ & 83.200 \\
\hline Jitt (\%) & 6.282 & 1.920 & -4.362 & $-69,44 \%$ & $-83,21 \%$ & 1.040 & 2.801 & 0.831 & -1.970 & $-70,33 \%$ & $-111,87 \%$ & 1.040 \\
\hline RAP (\%) & 3.597 & 1.158 & -2.439 & $-67,81 \%$ & $-83,61 \%$ & 0.680 & 1.774 & 0.510 & -1.264 & $-71,25 \%$ & $-115,54 \%$ & 0.680 \\
\hline PPQ (\%) & 3.899 & 1.056 & -2.843 & $-72,92 \%$ & $-92,94 \%$ & 0.840 & 1.634 & 0.492 & -1.142 & $-69,89 \%$ & $-143,83 \%$ & 0.840 \\
\hline sPPQ (\%) & 6.516 & 1.578 & -4.939 & $-75,80 \%$ & $-89,85 \%$ & 1.020 & 1.878 & 0.568 & -1.310 & $-69,76 \%$ & $-152,68 \%$ & 1.020 \\
\hline vf0 (\%) & 14.580 & 3.074 & -11.506 & $-78,92 \%$ & $-85,36 \%$ & 1.100 & 3.968 & 1.453 & -2.515 & $-63,38 \%$ & $-87,69 \%$ & 1.100 \\
\hline ShdB (dB) & 1.572 & 0.527 & -1.045 & $-66,48 \%$ & $-85,52 \%$ & 0.350 & 0.740 & 0.585 & -155 & $-20,95 \%$ & $-39,74 \%$ & 0.350 \\
\hline Shim (\%) & 13.412 & 5.584 & -7.828 & $-58,37 \%$ & $-81,52 \%$ & 3.810 & 8.390 & 6.573 & -1.817 & $-21,66 \%$ & $-39,67 \%$ & 3.810 \\
\hline APQ (\%) & 10.571 & 4.187 & -6.384 & $-60,39 \%$ & $-85,11 \%$ & 3.070 & 5.636 & 4.882 & -754 & $-13,38 \%$ & $-29,38 \%$ & 3.070 \\
\hline SAPQ (\%) & 19.268 & 6.399 & -12.869 & $-66,79 \%$ & $-85,58 \%$ & 4.230 & 7.429 & 6.980 & -449 & $-6,04 \%$ & $-14,04 \%$ & 4.230 \\
\hline $\operatorname{vAm}(\%)$ & 42.398 & 11.988 & -30.411 & $-71,73 \%$ & $-88,92 \%$ & 8.200 & 20.652 & 18.254 & -2.398 & $-11,61 \%$ & $-19,26 \%$ & 8.200 \\
\hline $\mathrm{NHR}$ & 0.413 & 0.247 & -166 & $-40,19 \%$ & $-74,44 \%$ & 0.190 & 0.217 & 0.152 & -65 & $-29,95 \%$ & $-240,74 \%$ & 0.190 \\
\hline VTI & 0.059 & 0.078 & 19 & $32,20 \%$ & $32,20 \%$ & 0.061 & 0.037 & 0.055 & 0.018 & $48,65 \%$ & $48,65 \%$ & 0.061 \\
\hline SPI & 12.581 & 9.120 & -3.461 & $-27,51 \%$ & $-27,51 \%$ & 14.120 & 16.684 & 15.152 & -1.532 & $-9,18 \%$ & $-59,75 \%$ & 14.120 \\
\hline FTRI (\%) & 2.724 & 0.633 & -2.091 & $-76,76 \%$ & $-117,87 \%$ & 0.950 & 0.579 & 0.049 & -530 & $-91,54 \%$ & $-91,54 \%$ & 0.950 \\
\hline ATRI (\%) & 11.523 & 3.301 & -8.222 & $-71,35 \%$ & $-114,95 \%$ & 4.370 & 3.914 & 2.651 & -1.263 & $-32,27 \%$ & $-32,27 \%$ & 4.370 \\
\hline DVB (\%) & 17.004 & 6.505 & -10.499 & $-61,74 \%$ & $-65,60 \%$ & 1.000 & 0.527 & 0.000 & -527 & $-100,00 \%$ & $-100,00 \%$ & 1.000 \\
\hline DSH (\%) & 0.000 & 0.000 & & & & 1.000 & 15.793 & 0.522 & -15.271 & $-96,69 \%$ & $-103,23 \%$ & 1.000 \\
\hline DUV (\%) & 40.223 & 12.222 & -28.001 & $-69,61 \%$ & $-71,39 \%$ & 1.000 & 0.696 & 0.173 & -523 & $-75,14 \%$ & $-75,14 \%$ & 1.000 \\
\hline NVB & 7 & 3,5 & $-3,5$ & $-50,00 \%$ & $-49,18 \%$ & 0.900 & 1 & 0 & -1 & $-100,00 \%$ & $-100,00 \%$ & 0.900 \\
\hline $\mathrm{NSH}$ & 0 & 0 & & & & 0.900 & 45 & 1.5 & $-43,5$ & $-96,67 \%$ & $-98,63 \%$ & 0.900 \\
\hline NUV & 90,5 & 27,5 & -63 & $-69,61 \%$ & $-69,92 \%$ & 0.900 & 2 & 0.5 & -15 & $-75,00 \%$ & $-136,36 \%$ & 0.900 \\
\hline
\end{tabular}

* a porcentagem de variação foi calculada tendo como valor referencial, o limiar de normalidade estabelecido pelo programa. Para os parâmetros que já estavam dentro da normalidade, o referencial foi o valor inicial.

† para o valor referencial, foi considerada a média de normalidade do programa, visto não constar um limiar definido.

$\S$ valor limite do tremor vocal, inserido na tabela (não consta no programa), que varia de $4-8 \mathrm{~Hz}{ }^{16}$.

$\mathrm{Hz}-\mathrm{Hertz}$

$\mathrm{dB}-$ Decibel

$\mu \mathrm{s}-$ microsegundo 
de ambos os sujeitos, apresentaram valores próximos (Tabela 3), indicando que o escape aéreo e ou a incoordenação pneumofonoarticulatória, ainda presentes ao final da terapia, foram similares para os dois casos estudados, podendo ser considerados peculiares da DP ${ }^{1,3-5}$.

Da mesma forma, a redução do $\mathrm{NRH}$ indicou menor componente de ruído presente à emissão. Observou-se que o sujeito 2 passou para o padrão de normalidade, provavelmente pelo decréscimo ocorrido na maioria das medidas, notando-se, também, que várias das medidas relacionadas ao ruído, às quebras e falhas de voz, e ao tremor alcançaram o padrão de normalidade (Jita, Jitt, RAP, PPQ, sPPQ, DVB, DSH, NVB, NUV, Fftr). O sujeito 1, apesar de ainda permanecer acima do limiar de normalidade, apresentou maior decréscimo absoluto do NHR, provavelmente pelas maiores mudanças, na variação absoluta, notadas nas medidas relacionadas ao jitter (Jita, Jitt, RAP, PPQ, sPPQ), shimmer (ShdB, Shim, APQ, sAPQ), tremor (FTRI e ATRI), variação de frequência e de amplitude (vfO e vAm) e de estabilidade (DVB, DUV, NUV).

A contração do músculo tireoaritenóideo é responsável pela adução e encurtamento das pregas vocais, e consequente redução da frequência fundamental. As medidas de redução da f0 e do STD, observadas neste estudo, sugerem que houve maior contração das pregas vocais, o que pode ter contribuído para a redução do escape aéreo e do nível de ruído, além de proporcionar maior estabilidade fonatória. As medidas de variação de frequência (vf0) e de amplitude (vAm), bem como as medidas DVB, DSH, DUV, NVB, NSH, NUV, mostram que houve maior estabilidade durante a fonação sustentada, que pode ser explicada, possivelmente, pela melhora da coordenação pneumofonoarticulatória, pelo maior suporte respiratório ${ }^{12}$, e pelo aumento da força glótica de adução ${ }^{10}$ promovidos pela terapia.

Observou-se, também, que mesmo os parâmetros que já se encontravam dentro do limiar de normalidade, antes da terapia, apresentaram redução nos seus valores, com exceção do VTI, variável que se relaciona ao ruído produzido pelo ar quando o fechamento das pregas vocais é incom- pleto ou fraco, que apresentou discreto aumento. No entanto, este discreto aumento do VTI, considerando-se a sua moderada consistência ${ }^{17}$, vai contra os demais resultados que, conjuntamente, sugerem a melhora da eficiência glótica de adução e redução do ruído após a terapia.

Os valores aumentados de Fftr e Fatr, encontrados apenas no sujeito 1 (Tabela 3), apesar de estarem dentro do padrão esperado para a DP ${ }^{16}$, podem estar relacionados à maior intensidade vocal obtida ao final da terapia, visto que o grau do tremor aumenta com o aumento da intensidade vocal. Este fato está possivelmente ligado à modificação da tensão muscular, nesta condição, que faz com que os músculos atuem mais rapidamente, aumentando a taxa de modulação ${ }^{20}$.

$\mathrm{O}$ aumento da intensidade vocal habitual, bem como da intensidade vocal forte (Tabela 4), pode ser reflexo das modificações ocorridas, tanto em nível fonatório como em nível respiratório, visto que o controle da intensidade é realizado por ambos os mecanismos, laríngeo e expiratório ${ }^{6,21}$, em resposta ao aumento da resistência glótica. A maior variação positiva no sujeito 1 , em ambas as situações, pode estar relacionada à maior modificação da coaptação glótica à videolaringoscopia, visto que o sujeito 2, por apresentar configuração de fechamento glótico completo pré-terapia, já mostrava valores maiores de intensidade no início da terapia. No entanto, o pequeno aumento de intensidade no sujeito 2 também sugere maior firmeza glótica de adução pós-terapia.

A intensidade vocal está relacionada à pressão subglótica, sendo diretamente proporcional às suas modificações ${ }^{6}$. Os fatores que contribuem para o aumento da pressão subglótica, e que, consequentemente, afetam a capacidade de produção de uma voz com intensidade adequada, são o fechamento glótico, a resistência glótica e o fluxo aéreo respiratório ${ }^{6,8}$.

$\mathrm{O}$ aumento da tonicidade laríngea e a melhor coaptação das pregas vocais aumentam a resistência glótica, proporcionando maior pressão subglótica ${ }^{22}$. Essas modificações fisiológicas podem melhorar também a qualidade vocal, visto que

Tabela 4 - Resultados da avaliação da intensidade vocal, nas intensidades habitual e forte, dos dois sujeitos

\begin{tabular}{lccc|ccc}
\hline Intensidade (dB) & $\begin{array}{c}\text { pré- } \\
\text { tratamento }\end{array}$ & $\begin{array}{c}\text { Sujeito 1 } \\
\text { pós- } \\
\text { tratamento }\end{array}$ & Variação & $\begin{array}{c}\text { Sujeito 2 } \\
\text { pré- } \\
\text { tratamento }\end{array}$ & $\begin{array}{c}\text { pós- } \\
\text { tratamento }\end{array}$ & \begin{tabular}{l} 
Variação \\
\hline Intensidade habitual
\end{tabular} \\
Intensidade forte & 68 & 62 & $6,90 \%$ & 61 & 63 & $3,29 \%$ \\
\hline
\end{tabular}

$\mathrm{dB}$ - Decibel 
aumentam o tempo em que as pregas vocais permanecem em contato (fase fechada do ciclo glótico), reduzindo o escape de ar e a presença de ruído à fonação, o que foi constatado em ambos os sujeitos deste estudo, após o período de terapia com instrumento de sopro.

Para valores de intensidade vocal, na literatura, foram encontrados, em indivíduos idosos saudáveis, valores variando de $67,4 \mathrm{~dB}$ a $84,8 \mathrm{~dB}$, nas condições de fraca e de forte intensidade, respectivamente ${ }^{23}$. As médias de intensidade vocal, para indivíduos adultos, foram de $63,46 \mathrm{~dB}$ em intensidade habitual e de $72,55 \mathrm{~dB}$ em intensidade forte ${ }^{22}$. E os valores médios de intensidade vocal, para homens com idade média de 59 anos, foram de $65,7 \mathrm{~dB}$ para intensidade fraca, de $73,4 \mathrm{~dB}$ para intensidade moderada e de $80,6 \mathrm{~dB}$ para forte intensidade ${ }^{21}$.

A variação na forma de captação e análise das medidas de intensidade vocal das pesquisas citadas anteriormente, não permite uma comparação direta deste parâmetro, com os dados do presente estudo, quanto ao padrão esperado de normalidade. No entanto, verificou-se que ambos os sujeitos deste trabalho mostraram valores de intensidade vocal habitual pré e pós-terapia semelhantes aos resultados de intensidade vocal fraca, em torno de $65,7 \mathrm{~dB}^{21}$ e de $67,4 \mathrm{~dB}{ }^{23}$, o que seria compatível com as características de DP. Os mesmos dados do presente estudo de casos, na condição de intensidade vocal habitual pré e pós-terapia, assemeIham-se aos de sujeitos adultos ${ }^{22}$, sugerindo que, mesmo com a DP, os participantes desta pesquisa apresentaram intensidade vocal habitual dentro de uma faixa ainda considerada normal.

Salienta-se que os valores de intensidade obtidos nesta pesquisa, para a voz habitual, aumentaram em ambos os sujeitos após a terapia, evidenciando mais uma vez, conforme já discutido anteriormente, o aumento da adução glótica e do maior controle respiratório promovidos pela mesma. Tais benefícios pós-terapia são reforçados pelos valores da voz em forte intensidade obtidos neste estudo, que se aproximam dos valores médios encontrados para indivíduos adultos ${ }^{22}$, ficando discretamente abaixo dos valores encontrados em outros estudos ${ }^{21,23}$, para a condição de forte intensidade vocal.
A diminuição da intensidade vocal é uma característica frequentemente encontrada na doença de Parkinson, que prejudica a inteligibilidade ${ }^{4}$ e a habilidade de comunicação. A terapia fonoaudiológica abrangendo a melhora da adução glótica e da respiração, por favorecer o aumento da intensidade vocal, reduz as dificuldades de comunicação dos indivíduos tratados.

Com relação à interferência do uso do medicamento, não existe um consenso na literatura com relação ao efeito do L-dopa nos parâmetros vocais e respiratórios. Desta forma, apesar da diferença no momento da avaliação respiratória e gravação das amostras de voz entre os dois sujeitos, o fator medicamento não foi considerado como fator interferente nas medidas realizadas ou na análise dos resultados.

Ao final do estudo constatou-se que, após a terapia com instrumento de sopro, as modificações respiratórias, acústicas e de intensidade vocal foram similares e positivas nos dois sujeitos deste estudo. No entanto, por tratar-se de um estudo piloto sugere-se a continuidade da pesquisa mantendo-se a metodologia, porém envolvendo maior número de sujeitos.

\section{CONCLUSÃO}

A partir da análise da relação entre os parâmetros estudados nos dois sujeitos pesquisados, pôde-se concluir que a terapia realizada com o instrumento de sopro pode proporcionar maior eficiência na adução glótica, além de melhor movimentação e controle respiratório, propiciando o uso mais efetivo do ar para fonação, com melhora da qualidade vocal, expressa pela redução das medidas de ruído, maior estabilidade fonatória e aumento da intensidade vocal.

\section{AGRADECIMENTOS}

Os autores agradecem ao médico otorrinolaringologista Dr. Rodrigo Ritzel pela colaboração e realização dos exames videolaringoscópicos. 


\begin{abstract}
Purpose: to check the phonatory function of Parkinson's disease patients before and after therapy with wind instrument emphasizing laryngeal, acoustic, respiratory and vocal intensity modifications. Methods: this research is a collective and prospective case study. Two subjects were submitted to larynx videoendoscopy, respiratory function valuation, recording of samples voice, vocal intensity measurement and therapy. The acoustic analysis were made through Multidimensional Voice Program advanced. Data analysis were weighed, once that the research is a collective case study. Results: the results showed that respiratory, acoustic and vocal intensity modifications were similar and positive in two subjects. Conclusion: the therapy carried through with wind instrument may provide higher efficiency in glottal adduction, movement and respiratory control, providing effective phonation breath use, vocal quality improvement with noise measures decrease, higher phonatory stability and vocal intensity increase.
\end{abstract}

KEYWORDS: Parkinson Disease; Speech Therapy; Voice

\section{REFERÊNCIAS}

1. Oguz H, Tunc T, Safak MA, Inan L, Kargin S, Demirci M. Objective voice changes in nondysphonic Parkinson's disease patients. J Otolaryngol. 2006; 35(5):349-54.

2. Dias AE. Características fonoarticulatórias na doença de Parkinson de início na meia idade e tardio [tese]. São Paulo (SP): Universidade de São Paulo; 2006.

3. CervantesO. Doençasneurológicas(repercussões laríngeas e vocais). In: Dedivitis RA, Barros APB. Métodos de avaliação e diagnóstico de laringe e voz. São Paulo: Lovise; 2002. p.145-75.

4. Silveira DN, Brasolotto AG. Reabilitação vocal em pacientes com doença de Parkinson: fatores interferentes. Pró-Fono. 2005; 17(2):241-50.

5. Sewall GK, Jiang J, Ford CN. Clinical evaluation of Parkinson's- related dysphonia. Laryngoscope. 2006; 116(10):1740-4.

6. Pinho SMR. Avaliação e tratamento da voz. In: Pinho SMR. Fundamentos em fonoaudiologia: tratando os distúrbios de voz. 2. ed. Rio de Janeiro: Guanabara Koogan; 2003. p.1-40.

7. Zhang Y, Jiang J, Rahn III DA. Studying vocal fold vibrations in Parkinson's disease with a nonlinear model. Chaos. 2005; 15(3):33903.

8. Sapienza CM, Davenport PW, Martin AD. Expiratory muscle training increases pressure support in high school band students. J Voice. 2002; 16(4):495-501.

9. Rahn III DA, Chou M, Jiang JJ, Zhang Y. Phonatory impairment in Parkinson's disease: evidence from nonlinear dynamic analysis and perturbation analysis. J Voice. 2007; 21(1):64-71.

10. Weikert M, Schlömicher-Their J. Laryngeal movements in saxophone playing: video-endoscopic investigations with saxophone players. A pilot study. J Voice. 1999; 13(2):265-73.

11. Eckley CA. Configuração glótica em tocadores de instrumento de sopro. Rev Bras Otorrinolaringol. 2006; 72(1):45-7.

12. Gallivan GJ, Eitnier CM. Vocal fold polyp in a professional brass/wind instrumentalist and singer. J Voice. 2006; 20(1):157-64.

13. Fritz C, Wolfe J. How do clarinet players adjust the resonances of their vocal tracts for different playing effects? J Acoust Soc Am. 2005; 118(5):3306-15.

14. Deniz O, Savci S, Tozkoparan E, Ince DI, Ucar M, Ciftci F. Reduced pulmonary function in wind instrument players. Arch Med Res. 2006; 37(4):506-10.

15. Alves-Mazzotti AJ. Usos e abusos dos estudos de caso. Cad Pesq. 2006; 36(129):637-51.

16. Titze IR. Workshop on acoustic voice analysis (Summary statement). lowa: National Center for Voice and Speech; 1994. 36p.

17. González J, Cervera T, Miralles JL. Análisis acústico de la voz: fiabilidad de un conjunto de parámetrosmultidimensionales. ActaOtorrinolaringol Esp. 2002; 53(4):256-68.

18. Saxon KG, Schneider CM. Vocal exercise physiology. San Diego: Singular Publishing; 1995. $157 p$.

19. Behlau M, Madazio G, Feijó D, Pontes P. Avaliação de voz. In: Behlau M. Voz: o livro do especialista. vol. 1. Rio de Janeiro: Revinter; 2001. p. 105-114.

20. Dromey C, Smith ME. Vocal tremor and vibrato in the same person: acoustic and electromyographic differences. J Voice. 2008; 22(5):541-5.

21. Makiyama K, Yoshihashi H, Mogitate M, Kida A. The role of adjustment of expiratory effort in the control of vocal intensity: clinical assessment of 
phonatory function. Otolaryngol Head Neck Surg. 2005; 132(4):641-6.

22. Koishi HU, Tsuji DH, Imamura R, Sennes LU. Variação da intensidade vocal: estudo da vibração das pregas vocais em seres humanos com videoquimografia. Rev Bras Otorrinolaringol. 2003; 69(4):464-70.

23. Hodge FS, Colton RH, Kelley RT. Vocal intensity characteristics in normal and elderly speakers. J Voice. 2001; 15(4):503-11.

RECEBIDO EM: 29/04/2008

ACEITO EM: 19/01/2009

Endereço para correspondência:

Juliana da Caldas Rosa

Rua Cel. Maurício Spalding de Souza, 419

Florianópolis - SC

CEP: 88035-110

E-mail: jucrosa@yahoo.com.br 